\title{
Laccase-Mediator System for the Elimination of Carbamazepine and Atrazine
}

\author{
Gülten Yüksek ${ }^{1,2}$, Lounes Haroune ${ }^{2}$, Didem Okutman Taş ${ }^{1}$, Hubert Cabana ${ }^{2}$ \\ ${ }^{1}$ Istanbul Technical University, Environmental Engineering Department \\ 34469 Maslak, Istanbul, Turkey \\ gulyuksek@itu.edu.tr; okutmand@itu.edu.tr \\ ${ }^{2}$ Groupe de recherche sur l'eau de l'Université de Sherbrooke, Département of Civil Engineering \\ Université de Sherbrooke \\ 2500 Boulevard de l'Université, Sherbrooke, Québec J1K 2R1, Canada \\ Gulten.Yuksek@Usherbrooke.ca; Lounes.Haroune@Usherbrooke.ca; Hubert.Cabana@Usherbrooke.ca
}

\section{Extended Abstract}

The inefficiency of conventional wastewater treatment plants (WWTPs) to remove organic contaminants raised concerns. Consequently, new techniques such as enzymatic processes gained interest. While oxidative enzyme laccase represents a potentially attractive new eco-friendly treatment, many organic contaminants are persistent to laccase oxidation. The utilization of the so-called laccase-mediator system was the only option for increasing the oxidation potential of these enzymes.

The aim of this study was to determine the whole potential of laccase enzyme for carbamazepine (CBZ) and atrazine (ATZ) removal using different mediators. The first sets of experiments were carried out at environmental concentration $\left(150 \mu \mathrm{g} \mathrm{l}^{-1}\right)$ with a single and mixed organic contaminants solution, CBZ and ATZ. Laccase used in the experiment was partly purified from Pleurotus dryinus strain. Different kinds of mediators (acetophenone, 2,2'-Azino-bis(3ethylbenzothiazoline-6-sulfonic acid (ABTS), 2,2,6,6-Tetramethylpiperidin-1-yl)oxyl (TEMPO) and the phenolic organic contaminant acetaminophen) were evaluated. The removal efficiency, laccase activity and $\mathrm{pH}$ was monitored. The results showed that CBZ and ATZ were roughly removed (-100\%) after $36 \mathrm{~h}$ in individual compound solution. However, ATZ and CBZ were not removed by laccase alone, suggesting the need of using mediator system. In a second set of experiments, the mix of ATZ and CBZ were significant slightly lower with removal up to $66 \%$.

The results showed that laccase-mediator systems were very efficient to remove recalcitrant organic contaminants such as ATZ and CBZ in individual compound solutions even at environmentally relevant concentrations. However, the binary mixture significantly decreased the removal efficiency, suggesting that the composition of the solution becomes a principal factor. Removal efficiency of ATZ and CBZ in single compound solutions sets were observed up to $100 \%$ during the first $36 \mathrm{~h}$ and no significant difference was observed for all the tested mediators. Regardless of single compounds sets, ATZ and CBZ in mixed compounds sets removal were less than $65 \%$.

\section{Acknowledgements}

This study was funded by the Scientific and Technological Research Council of Turkey (TUBITAK) as well as the Natural Science and Engineering Research Council of Canada (NSERC) grant programs. Also, the authors would want to thank Olivier Savary and Sabrina Saibi for their technical support. 